\title{
Effects of ketamine on lipopolysaccharide-induced depressive-like behavior and the expression of inflammatory cytokines in the rat prefrontal cortex
}

\author{
CHUN YANG $^{1}$, JIANG SHEN ${ }^{1}$, TAO HONG ${ }^{1}$, TING-TING HU ${ }^{1}$, ZHANG-JUN LI ${ }^{1}$, \\ HAI-TAO ZHANG ${ }^{1}$, YONG-JUN ZHANG ${ }^{1}$, ZHI-QIANG ZHOU ${ }^{2}$ and JIAN-JUN YANG ${ }^{2}$ \\ ${ }^{1}$ Department of Anesthesiology, The Third Affiliated Hospital of Soochow University, \\ Changzhou, Jiangsu 213003; ${ }^{2}$ Department of Anesthesiology, Jinling Hospital, School of Medicine, \\ Nanjing University, Nanjing, Jiangsu 210002, P.R. China
}

Received March 6, 2013; Accepted July 11, 2013

DOI: $10.3892 / \mathrm{mmr} .2013 .1600$

\begin{abstract}
The present study was designed to investigate the effects of ketamine on lipopolysaccharide (LPS)-induced depressive-like behavior and the expression of inflammatory cytokines in the rat prefrontal cortex. Thirty male Wistar rats were randomly divided into 3 groups $(n=10)$ : saline group (S group), LPS only group (L group) and LPS plus ketamine group (LK group). A forced swimming test (FST) was performed. On the first day, rats were placed into water for $15 \mathrm{~min}$. Twenty-four hours later, rats were treated again as in the first test for a $5 \mathrm{~min}$ session, and the immobility time was recorded. The prefrontal cortex was harvested for the determination of the interleukin (IL)-1 $\beta$, IL-6 and IL-10 levels. Compared with the S group, rats in the L group had significantly increased immobility time in the FST and expression of IL-1 $\beta$ and IL-6, and significantly decreased expression of IL-10 in the prefrontal cortex $(\mathrm{P}<0.05)$. However, rats in the LK group had significantly decreased immobility times in the FST and expression of IL- $1 \beta$ and IL- 6 , and significantly increased expression of IL-10 in the prefrontal cortex compared with the $\mathrm{L}$ group $(\mathrm{P}<0.05)$. Ketamine can alleviate LPS-induced depressive-like behavior, and its effect is likely associated with changes in the expression of inflammatory cytokines in the rat prefrontal cortex.
\end{abstract}

\section{Introduction}

Ketamine is a classical anesthetic agent that is widely used in clinical surgery. Recently, it has been validated by mounting

Correspondence to: Professor Jian-Jun Yang, Department of Anesthesiology, Jinling Hospital, School of Medicine, Nanjing University, 305 East Zhongshan Road, Nanjing, Jiangsu 210002, P.R. China

E-mail: hippocampus@sohu.com

Key words: ketamine, depressive-like behavior, lipopolysaccharide, inflammatory cytokines, prefrontal cortex studies demonstrating that ketamine has a robust therapeutic effect for the treatment of depression (1-5). However, the underlying mechanisms involved remain to be elucidated.

At present, a large number of theories regarding the underlying mechanisms of depression have been reported, but no study has elucidated the mechanisms clearly. It has been shown that the expression of pro-inflammatory cytokines in the plasma of depressed patients is significantly increased as compared with controls (6-8). Moreover, several lines of evidence have indicated that the expression of pro-inflammatory cytokines in the peripheral blood of patients tends to return to normal levels after treatment with antidepressant agents $(9,10)$. Several lines of evidence have indicated that a single dose of lipopolysaccharide (LPS) administered intraperitoneally in rodents is capable of eliciting depressive-like behavioral deficits $(11,12)$. Collectively, these findings indicate that inflammatory cytokines are most likely implicated in the pathogenesis of depression.

Ketamine is often recommended for use in surgery in sepsis patients due to its anti-inflammatory effects $(13,14)$. However, to date, no information has been available in the literature on the association between the antidepressant effects of ketamine and the expression of inflammatory cytokines in brain tissue. Therefore, based on this point, we considered whether the modulation of inflammatory cytokines is the underlying mechanism involved in exerting the antidepressant effects of ketamine, and furthermore hypothesized that the changes in inflammatory cytokines were most likely implicated in the underlying mechanisms of ketamine-elicited therapeutic effects for depression (15). Thus, in the present study, we observed the effects of ketamine on LPS-induced depressive-like behavior, and determined the levels of IL-1 $\beta$, IL-6 and IL-10 in the prefrontal cortex in a rat model.

\section{Materials and methods}

Animals. Thirty male Wistar rats (200-300 g body weight) were purchased from the Shanghai Animal Center, Shanghai, China. The animals were housed 5 per cage with food and water available ad libitum, and were maintained on a $12 \mathrm{~h}$ 
light/dark cycle (lights on at 7:00 am). Animals were involved in this experiment in accordance with the Guide for Care and Use of Laboratory Animals of Nanjing University (Nanjing, Jiangsu, China). The study was approved by the Ethics Committee of The Third Affiliated Hospital of Soochow University (Changzhou, China).

Drugs and interventions. Ketamine was purchased from Gutian Pharmaceutical Co. (Fujian, China). LPS was purchased from Sigma Co. (St. Louis, MO, USA). Rats were randomly divided into three groups ( $\mathrm{n}=10$ each): saline group (S group), LPS only group (L group) and LPS plus ketamine group (LK group). All rats in the three groups were forced to swim for $15 \mathrm{~min}$ on the first day. Twenty-two hours later, rats were intraperitoneally injected with saline or LPS $(1 \mathrm{mg} / \mathrm{kg})$. One hour later, rats were intraperitoneally injected with saline or ketamine $(10 \mathrm{mg} / \mathrm{kg})$ in the same volume. Another $1 \mathrm{~h}$ later, the forced swimming test (FST) was carried out for $5 \mathrm{~min}$ and the immobility time was recorded. Immediately after the FST, the rats were sacrificed and the prefrontal cortex was dissected and stored at $-80^{\circ} \mathrm{C}$ for biochemical analyses.

FST. In accordance with a previous study (16), this test included two individual stimuli to a cylindrical tank with water in which the rats cannot touch the bottom of the tank. The volume of the tank was $60 \mathrm{~cm}$ tall, $30 \mathrm{~cm}$ in diameter, and was filled with water to a depth of $40 \mathrm{~cm}$. Water in the tank was changed after the testing of every rat. All animal behavioral observation and other procedures were carried out between 9:00 am and 15:00 pm. First, rats were placed in the water for 15 min without exposure to any drug. Twenty-four hours later, rats were treated again as in the first test for a 5 min session, and the immobility time was recorded. The definition of immobility in the FST was that the rat remained floating in the water without struggling and only made movements necessary to keep its head above the water. Assessment of immobility time was carried out by the same trained observer.

Testing IL-1 $\beta$ levels. The prefrontal cortex was dissected and homogenized in a solution containing $0.32 \mathrm{M}$ sucrose, $20 \mathrm{mM}$ HEPES (pH 7.4), 1 mM EDTA, 1X protease inhibitor cocktail, $5 \mathrm{mM} \mathrm{NaF}$ and $1 \mathrm{mM}$ sodium vanadate. The homogenate was centrifuged for $10 \mathrm{~min}$ at $870 \mathrm{x} \mathrm{g}$ at $4^{\circ} \mathrm{C}$. The pellet (nuclear fraction) contained nuclei and large cell debris. The supernatant was centrifuged at $16,000 \times \mathrm{g}$ for $10 \mathrm{~min}$. After centrifugation, the supernatant (cytosolic fraction) was removed and the pellet (crude synaptosomal fraction) was resuspended and sonicated in protein lysis buffer [50 mM Tris- $\mathrm{HCl}(\mathrm{pH} 7.5)$, $150 \mathrm{mM} \mathrm{NaCl}, 1 \%$ Triton X-100, 0.1\% SDS, 2 mM EDTA, $1 \mathrm{mM} \mathrm{NaVO}_{3}, 5 \mathrm{mM} \mathrm{NaF}$ and $1 \mathrm{X}$ protease inhibitor cocktail]. Protein concentration was determined by $\mathrm{ABC}$ protein assay. For western blotting, equal amounts of proteins (10-20 $\mu \mathrm{g})$ for each sample were loaded into 10-15\% SDS PAGE gels for electrophoresis. Polyvinylidene difluoride (PVDF) membranes (Nanjing Jiancheng Bioengineering Company, Nanjing, China) with transferred proteins were blocked with $2 \%$ BSA (Equitech-Bio, Inc., Kerrville, TX, USA) in PBST phosphate buffer solution [PBS (Sigma-Aldrich Canada, Oakville, ON, Canada) $+0.1 \%$ Tween-20] for $1 \mathrm{~h}$ and kept with primary antibodies overnight at $4^{\circ} \mathrm{C}$. The primary antibody (anti-IL-1 $\beta$ ) was used. The next day, blots were washed three times in PBST and incubated with horseradish peroxidase-conjugated anti-mouse or anti-rabbit secondary antibody $(1: 5,000-1: 10,000)$ for $1 \mathrm{~h}$. After three final washes with PBST, bands were detected using enhanced chemiluminescence (ECL; Beyotime Company, Nantong, China). The blots were then incubated in stripping buffer (2\% SDS, $100 \mathrm{mM} \beta$-mercaptoethanol, $50 \mathrm{mM}$ Tris $\mathrm{pH}$ 6.8) for $30 \mathrm{~min}$ at $50-55^{\circ} \mathrm{C}$ followed by three washes with PBST. The stripped blots were kept in blocking solution for $1 \mathrm{~h}$ and incubated with the primary antibody directed against total levels of $\beta$-actin as the loading control. Densitometric analysis of immunoreactivity for each protein was conducted using Image $\mathbf{J}$ software (National Institutes of Health, Bethesda, MD, USA).

Testing IL-6 and IL-10 levels. IL-6 and IL-10 levels in the prefrontal cortex were individually measured by anti-IL-6 and anti-IL-10 sandwich-ELISA according to the manufacturer's instructions (Chemicon, Billerica, MA, USA). Rat hippocampus was homogenized in PBS with $1 \mathrm{mM}$ phenylmethylsulfonyl fluoride (PMSF) and 1 mM EGTA. Microtiter plates (48-well flat-bottom) were coated for $24 \mathrm{~h}$ with the samples diluted 1:2 in sample diluent and the standard curve ranged from 7.8 to $500 \mathrm{pg} / \mathrm{ml}$ of IL- 6 and IL-10. The plates were then washed four times with sample diluent and a monoclonal anti-IL-6 and anti-IL-10 rabbit antibody diluted 1:1,000 in sample diluent was added to each well and incubated for $3 \mathrm{~h}$ at room temperature. After washing, a peroxidaseconjugated anti-rabbit antibody (diluted 1:1,000) was added to each well and incubated at room temperature for $1 \mathrm{~h}$. After addition of streptavidin-enzyme, substrate and stop solution, the amount of IL- 6 and IL-10 were determined by absorbance at $450 \mathrm{~nm}$, respectively. The standard curve demonstrates a direct relationship between optical density (OD) and IL-6 or IL-10 concentration. Total protein was measured by Lowry's method using bovine serum albumin as a standard.

Statistical analysis. Data are expressed as the means \pm SD. Statistical analyses were made by one-way analysis of variance (ANOVA) and post hoc analyses were performed by least significant difference (LSD) tests. These statistical analyses were conducted by Statistical Product for Social Sciences (SPSS version 17.0; SPSS Inc., Chicago, IL, USA). P $<0.05$ was considered to indicate a statistically significant result.

\section{Results}

Immobility time of rats in the FST. The immobility time of the rats during the FST showed significant differences among the three groups $[F(2,27)=16.44, \mathrm{P}<0.05]$. Administration of LPS only showed a significant increase in the immobility time as compared with saline only $(\mathrm{P}<0.05)$. Moreover, administration of LPS plus ketamine significantly increased the immobility time as compared with LPS only $(\mathrm{P}<0.05 ;$ Fig. 1).

Expression of IL-1 $\beta$ in the rat prefrontal cortex. The expression of IL-1 $\beta$ in the rat prefrontal cortex showed significant differences among these groups $[\mathrm{F}(2,27)=32.13, \mathrm{P}<0.01]$. Compared with saline only, administration of LPS only showed a significant increase in the IL-1 $\beta$ levels $(\mathrm{P}<0.01)$, while administration 


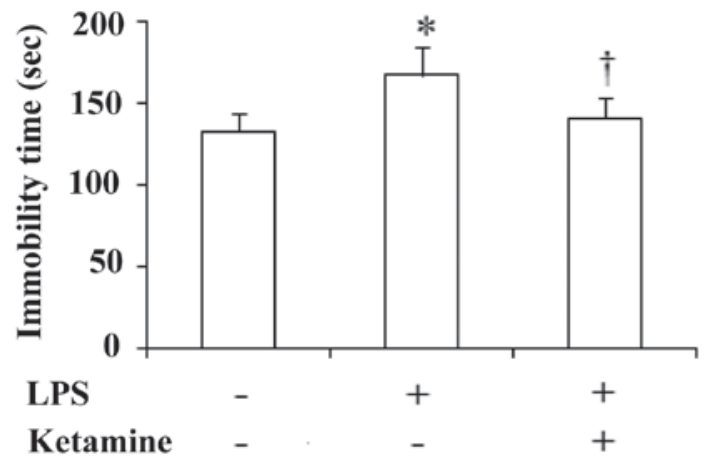

Figure 1. Immobility time (means $\pm \mathrm{SD}$ ) of rats $(\mathrm{n}=10)$ in the forced swimming test. LPS administered alone showed a significant increase in the immobility time as compared with the saline group $\left({ }^{*} \mathrm{P}<0.05\right)$. Moreover, administration of LPS plus ketamine significantly decreased the immobility time as compared with LPS only ( $\mathrm{P}<0.05)$. LPS, lipopolysaccharide.
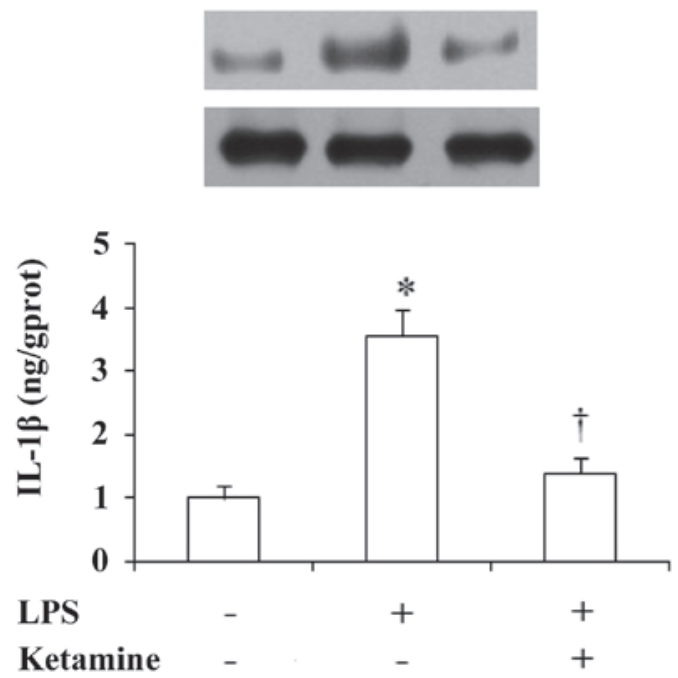

Figure 2. Expression of IL-1 $\beta$ (means \pm SD) in the rat prefrontal cortex $(n=3)$ Administration of LPS alone showed a significant increase in the expression of IL- $1 \beta$ in the rat prefrontal cortex as compared with the saline group $\left({ }^{*} \mathrm{P}<0.01\right)$. Moreover, administration of LPS plus ketamine significantly decreased the expression of IL-1 $\beta$ as compared with LPS only ( $(\mathrm{P}<0.01)$. IL, interleukin; LPS, lipopolysaccharide.

of LPS plus ketamine significantly decreased the expression of IL-1 $\beta$ as compared with LPS only $(\mathrm{P}<0.01$; Fig. 2$)$.

Expression of IL- 6 in the rat prefrontal cortex. The expression of IL-6 in the rat prefrontal cortex showed significant differences among these groups $[\mathrm{F}(2,27)=8.36, \mathrm{P}<0.05]$. Compared with saline only, administration of LPS only showed a significant increase in the IL-6 levels $(\mathrm{P}<0.05)$. Compared with LPS only, administration of LPS plus ketamine significantly decreased the IL-6 levels $(\mathrm{P}<0.05$; Fig. 3$)$.

Expression of IL-10 in the rat prefrontal cortex. The expression of IL-10 in the rat prefrontal cortex showed significant differences among the three groups $[\mathrm{F}(2,27)=6.83, \mathrm{P}<0.05]$. Compared with saline only, administration of LPS only showed a significant decrease in the IL-10 levels $(\mathrm{P}<0.05)$, while administration of LPS plus ketamine significantly increased the IL-10 levels as compared with LPS only ( $\mathrm{P}<0.05$; Fig. 4).

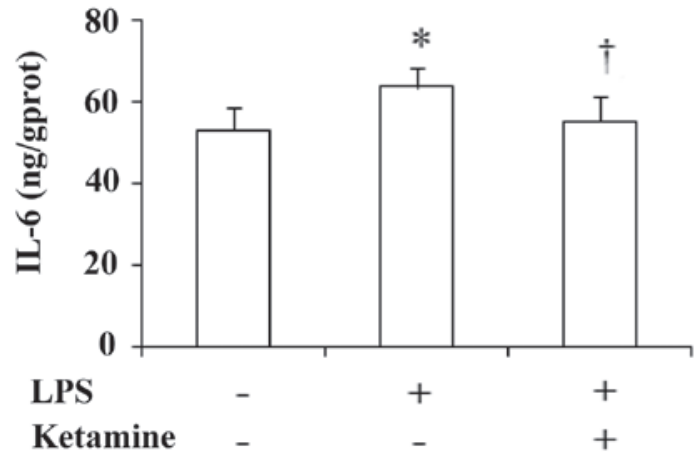

Figure 3. Expression of interleukin IL-6 (means \pm SD) in the rat prefrontal cortex $(n=10)$. Administration of LPS alone showed a significant increase in the expression of IL- 6 in the rat prefrontal cortex as compared with the saline group $\left({ }^{*} \mathrm{P}<0.05\right)$. Moreover, administration of LPS plus ketamine significantly decreased the expression of IL-6 as compared with LPS only $(\mathrm{P}<0.05)$. IL, interleukin; LPS, lipopolysaccharide.

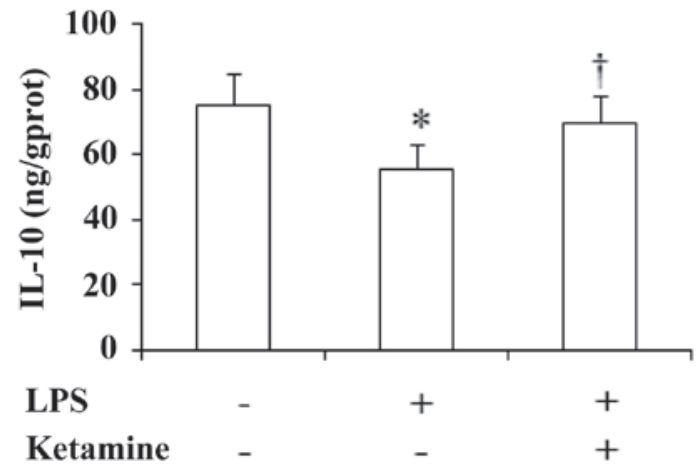

Figure 4. Expression of interleukin IL-10 (means \pm SD) in the rat prefrontal cortex $(n=10)$. Administration of LPS alone showed a significant decrease in the expression of IL-10 in the rat prefrontal cortex as compared with the saline group $\left({ }^{*} \mathrm{P}<0.05\right)$. Moreover, administration of LPS plus ketamine significantly increased the expression of IL-10 as compared with LPS only ('P<0.05). IL, interleukin; LPS, lipopolysaccharide.

\section{Discussion}

The results of the present study demonstrated that ketamine significantly attenuated the LPS-induced increase of the immobility time of rats during the FST, and significantly decreased the expression of IL- $1 \beta$ and IL- 6 in the rat prefrontal cortex. The results also showed downregulated expression of IL-10 in the rat prefrontal cortex. These findings indicate that ketamine has antidepressant effects in the rat model of LPS-induced depressive-like behavior. Li et al (5) have reported that ketamine administered at a sub-anesthetic dose of $10 \mathrm{mg} / \mathrm{kg}$ exerts antidepressant effects in rats during the FST. Therefore, we used ketamine at a dose of $10 \mathrm{mg} / \mathrm{kg}$ to observe its antidepressant effect in the present study, and the result was consistent with the previous findings (5). The only difference in the present study is that we adopted a novel depressive-like rat model, which was induced by intraperitoneal injection of LPS. Although the antidepressant effect of ketamine has been reported and validated in previous studies, the present study is the first to elucidate its effect for the treatment of depression in the LPS-induced depressive-like rat model $(1,3,4)$. 
LPS is an element in the Gram-negative bacterial wall that may cause fever, microcirculation disturbance, shock and disseminated intravascular coagulation $(17,18)$. It has been shown that LPS has the potential to induce depressive-like behavior in a rat model (19). In this study, we observed that LPS administered at a dose of $1 \mathrm{mg} / \mathrm{kg}$ significantly increased the immobility time of rats during the FST, which suggested that the depressive-like rat model was successfully constructed. However, Kang et al (20) have indicated that mice exhibit depressive-like manifestations after intraperitoneal injection of LPS at the dose of $0.8 \mathrm{mg} / \mathrm{kg}$. The discrepancy between the administration doses of LPS is most likely due to the species difference. Moreover, another study carried out by Hosseini et al (11) using LPS at the dose of $100 \mu \mathrm{g} / \mathrm{kg}$ induced depressive-like behavioral deficits in Wistar rats, but we did not get the same result in our pilot experiment (data not shown).

Ketamine is widely used for surgery in patients with sepsis due to its anti-inflammatory effect. A previous study has reported that ketamine has a fast-acting anti-inflammatory effect (21). Moreover, ketamine exerts a rapid antidepressant effect within 1-2 $\mathrm{h}$ after administration. The coincidence of the fast-acting antidepressant and anti-inflammatory effects suggests that modulating inflammatory cytokines are likely to participate in the antidepressant effect of ketamine. As mentioned previously, pro-inflammatory cytokines were reversed to normal levels after treatment with antidepressant agents $(9,10)$. Therefore, we demonstrated that LPS-induced behavioral deficits and changes in expression of inflammatory cytokines were reversed after ketamine administration. Consequently, the hypothesis we proposed was verified in the present study.

Given the preclinical and clinical data indicating elevations in IL-1 $\beta$ and IL- 6 levels in plasma and/or the brain of patients or animals with depression $(8,19)$, we were intrigued to evaluate IL-1 $\beta$ and IL- 6 protein levels in the prefrontal cortex in a rat model demonstrating depressive-like behaviors following LPS exposure. Notably, analysis of IL-1 $\beta$ and IL-6 levels after ketamine treatment alleviated LPS-induced depressive-like behavior and was associated with the downregulation of IL-1 $\beta$ and IL- 6 levels. These results as well as the changed expression of IL-10 revealed that these three important inflammatory cytokines were implicated in the mechanisms involved in the antidepressant effect of ketamine. Furthermore, the mediation of the inflammatory pathway in the pathogenesis and the therapeutic mechanisms of depression has been validated again in this study.

However, a limitation for this study is that we did not conduct a ketamine only group, since ketamine only was not suitable for the study design and the result has been observed in our previous studies $(22,23)$. In conclusion, the antidepressant effect of ketamine was associated with the modulation of inflammatory cytokines. Future studies are required to further elucidate the relationship between the inflammation pathway and the antidepressant effect of ketamine.

\section{Acknowledgements}

This study was supported by a grant from the National Natural Science Foundation of China (no. 30872424).

\section{References}

1. Aan Het Rot M, Zarate CA Jr, Charney DS and Mathew SJ: Ketamine for depression: where do we go from here? Biol Psychiatry 72: 537-547, 2012.

2. Mathew SJ, Shah A, Lapidus K, et al: Ketamine for treatmentresistant unipolar depression: current evidence. CNS Drugs 26: 189-204, 2012.

3. Machado-Vieira R, Salvadore G, Diazgranados N and Zarate CA Jr: Ketamine and the next generation of antidepressants with a rapid onset of action. Pharmacol Ther 123: 143-150, 2009.

4. Krystal JH: Ketamine and the potential role for rapid-acting antidepressant medications. Swiss Med Wkly 137: 215-216, 2007.

5. Li N, Lee B, Liu RJ, et al: mTOR-dependent synapse formation underlies the rapid antidepressant effects of NMDA antagonists. Science 329: 959-964, 2010.

6. Catena-Dell'Osso M, Bellantuono C, Consoli G, Baroni S, Rotella F and Marazziti D: Inflammatory and neurodegenerative pathways in depression: a new avenue for antidepressant development? Curr Med Chem 18: 245-255, 2011.

7. Hayley S, Poulter MO, Merali Z and Anisman H: The pathogenesis of clinical depression: stressor- and cytokine-induced alterations of neuroplasticity. Neuroscience 135: 659-678, 2005.

8. Raedler TJ: Inflammatory mechanisms in major depressive disorder. Curr Opin Psychiatry 24: 519-525, 2011.

9. De Berardis D, Conti CM, Serroni N, et al: The effect of newer serotonin-noradrenalin antidepressants on cytokine production: a review of the current literature. Int J Immunopathol Pharmacol 23: 417-422, 2010.

10. Song $\mathrm{C}$ and Wang $\mathrm{H}$ : Cytokines mediated inflammation and decreased neurogenesis in animal models of depression. Prog Neuropsychopharmacol Biol Psychiatry 35: 760-768 2011.

11. Hosseini M, Zakeri S, Khoshdast S, et al: The effects of Nigella sativa hydro-alcoholic extract and thymoquinone on lipopolysaccharide-induced depression like behavior in rats. J Pharm Bioallied Sci 4: 219-225, 2012.

12. Park SE, Lawson M, Dantzer R, Kelley KW and McCusker RH: Insulin-like growth factor-I peptides act centrally to decrease depression-like behavior of mice treated intraperitoneally with lipopolysaccharide. J Neuroinflammation 8: 179, 2011

13. Loix S, De Kock M and Henin P: The anti-inflammatory effects of ketamine: state of the art. Acta Anaesthesiol Belg 62: 47-58, 2011.

14. Sun J, Zhou ZQ, Lv R, Li WY and Xu JG. Ketamine inhibits LPS-induced calcium elevation and NF-kappa B activation in monocytes. Inflamm Res 53: 304-308, 2004.

15. Yang JJ, Zhou ZQ and Yang C: Letter to the editor: does ketamine exert a fast-acting antidepressant effect via inhibition of pro-inflammatory cytokines? Psychol Med 41: 1787, 2011.

16. Porsolt RD, Le Pichon M and Jalfre M: Depression: a new animal model sensitive to antidepressant treatments. Nature 266: 730-732, 1977.

17. Herzum I and Renz H: Inflammatory markers in SIRS, sepsis and septic shock. Curr Med Chem 15: 581-587, 2008.

18. Biswas SK and Lopez-Collazo E: Endotoxin tolerance: new mechanisms, molecules and clinical significance. Trends Immunol 30: 475-487, 2009.

19. Dunn AJ, Swiergiel AH and de Beaurepaire R: Cytokines as mediators of depression: what can we learn from animal studies? Neurosci Biobehav Rev 29: 891-909, 2005.

20. Kang A, Hao H, Zheng X, et al: Peripheral anti-inflammatory effects explain the ginsenosides paradox between poor brain distribution and anti-depression efficacy. J Neuroinflammation 8: 100, 2011.

21. Taniguchi $\mathrm{T}$ and Yamamoto $\mathrm{K}$ : Anti-inflammatory effects of intravenous anesthetics on endotoxemia. Mini Rev Med Chem 5: 241-245, 2005.

22. Yang C, Li WY, Yu HY, et al: Tramadol pretreatment enhances ketamine-induced antidepressant effects and increases mammalian target of rapamycin in rat hippocampus and prefrontal cortex. J Biomed Biotechnol 2012: 175619, 2012.

23. Yang C, Hong T, Shen J, et al: Ketamine exerts antidepressant effects and reduces IL-1 $\beta$ and IL- 6 levels in rat prefrontal cortex and hippocampus. Exp Ther Med 5: 1093-1096, 2013. 